\title{
Diversidade e abundância de aves costeiras em um trecho do litoral norte do Rio Grande do Sul, Brasil
}

\author{
Andressa Muller * \\ Marcelo Pereira de Barros \\ Universidade Feevale, Rodovia RS 239, km 2755, CEP 93352-000, Novo Hamburgo - RS, Brasil \\ * Autor para correspondência \\ dessamuller@terra.com.br
}

Submetido em 07/05/2012

Aceito para publicação em 25/06/2013

\section{Resumo}

A avifauna litorânea é um importante elemento na dinâmica dos ecossistemas onde vive, sendo a planície costeira considerada uma área de altíssima prioridade para a conservação da biodiversidade regional. Devido à carência de informações sobre as aves que frequentam o litoral norte do Rio Grande do Sul, principalmente em trechos de intensa ocupação antrópica, e a importância desse grupo como um constituinte da biodiversidade, este artigo objetiva, com vistas à conservação, fornecer dados sobre a avifauna costeira. $\mathrm{O}$ estudo apresenta um levantamento das aves marinhas e costeiras, em um trecho entre os municípios de Tramandaí e Palmares do Sul, no litoral norte do estado, estabelecendo a variação sazonal e estimando a abundância de cada espécie ao longo de um ano. $O$ estudo foi realizado em uma extensão de $50 \mathrm{~km}$ de beira de praia, percorrida mensalmente com um automóvel, e as aves observadas nesse trajeto foram contabilizadas com auxílio de binóculo. As amostragens ocorreram durante um período de 14 meses. Foram registradas 21.888 aves, de 44 espécies, pertencentes a 20 famílias, que representam 10 ordens. As famílias mais abundantes foram Scolopacidae, Charadriidae, Laridae e Haematopodidae, representantes da ordem Charadriiformes.

Palavras-chave: Aves marinhas; Sul do Brasil; Zona costeira

\section{Abstract}

Diversity and abundance of marine birds in a stretch of the north coast of Rio Grande do Sul, Brazil. The coastal avifauna is an important element in the dynamics of the ecosystems where it lives, and the coastal plain is regarded as a very high priority area for preserving the regional biodiversity. Due to the lack of information on the birds which go to the north coast of Rio Grande do Sul, especially in stretches with an intense anthropic occupation, and the importance of this group as a biodiversity constituent, this paper aims, with a conservational purpose, to provide data on the coastal avifauna. The study presents a survey of marine and coastal birds, in a stretch between the towns of Tramandai and Palmares do Sul, in the state's northern coast, establishing the seasonal variation and estimating the abundance of each species throughout one year. The study took place over $50 \mathrm{~km}$ of seashore, monthly went through by car, and the birds observed in this route were counted with the aid of binoculars. The samplings took place within the period of 14 months. One recorded 21,888 birds, of 44 species, belonging to 20 families, which represent 10 orders. The most abundant families were Scolopacidae, Charadriidae, Laridae, and Haematopodidae, representing the Charadriiformes order.

Key words: Coastal zone; Marine birds; Southern Brazil 


\section{Introdução}

A América do Sul apresenta uma das mais ricas biodiversidades da Terra, contudo, o conhecimento específico não chega a $10 \%$ da riqueza do planeta. O Brasil é considerado o país das aves (AZEVEDO JUNIOR et al., 2004), abrigando uma grande parcela da biodiversidade das aves marinhas e costeiras do mundo (VOOREN; BRUSQUE, 1999). Segundo o Comitê Brasileiro de Registros Ornitológicos (CBRO, 2011), 1832 espécies de aves ocorrem no território brasileiro dentre as quais 661 espécies são encontradas no Rio Grande do Sul (BENCKE et al., 2010). Para o litoral norte do estado foram registradas, até o momento, 57 espécies de aves costeiras (COSTA; SANDER, 2008).

As zonas costeiras, incluindo o litoral brasileiro, foram afetadas nas últimas décadas pela degradação ambiental desencadeada através do rápido crescimento demográfico e suas consequentes alterações nos ambientes litorâneos, além das crescentes demandas econômicas, que causaram declínio dos recursos naturais (SEELIGER et al., 1998; EFE, 2004).

A costa do Rio Grande do Sul, compreendida entre o Rio Mampituba e o arroio Chuí estende-se por $620 \mathrm{~km}$ (BELTON, 2000) e varia de $10 \mathrm{~km}$ de largura mais a norte até $100 \mathrm{~km}$ próximo a lagoa dos Patos (RAMBO, 2000), caracterizando-se como a planície costeira mais ampla do país (ABSALONSEN; TOLDO JR., 2007). Segundo o Programa de Gerenciamento Costeiro do Rio Grande do Sul (GERCO, 1988) o litoral do estado pode ser dividido em três regiões: norte, médio e sul. De acordo com Calliari et al. (2005), a região do litoral norte estende-se do município de Torres até o Farol Berta, ao sul do balneário de Dunas Altas, localizado no município de Palmares do Sul.

Brack (2006) destaca que os principais balneários do estado concentram-se no compartimento da planície costeira do litoral norte, ocupando, densamente, a faixa de dunas e campos litorâneos entre a praia e o cordão de lagoas. De acordo com o Projeto RS Biodiversidade a planície costeira é considerada uma área de altíssima prioridade para a conservação da biodiversidade, com significado a nível mundial (SEMA, 2007), pois a avifauna encontra nesta região, preservada e com baixo nível de perturbação, recursos alimentares, repouso e abrigo necessários para sua sobrevivência e condicionamento para a migração (VOOREN; BRUSQUE, 1999).

A escassez e a indisponibilidade de informações técnicas sobre a fauna de uma região tem sido um dos fatores responsáveis pela dificuldade no direcionamento de esforços conservacionistas. Informações adequadas e confiáveis podem contribuir para a identificação de áreas de alto interesse para a conservação da biodiversidade regional antes que sejam afetadas pela expansão urbana, agrícola e industrial desordenada (MAURÍCIO; DIAS, 2001).

Poucos são os estudos sobre aves costeiras no litoral norte do estado, o que indica uma lacuna de informações sobre a avifauna dessa região. Costa e Castro (2007) monitoraram as espécies das ordens Falconiformes e Cathartiformes no trecho de Imbé a Torres, assim como Costa e Sander (2008), que verificaram a variação sazonal das ordens Charadriiformes e Ciconiiformes no mesmo trecho. Franz et al. (2008) registraram dois indivíduos de atobá (Sula dactylatra) no litoral norte, sendo estes os primeiros registros para o Rio Grande do Sul. Accordi (2008) comparou os padrões de riqueza, composição e abundância de aves entre os balneários de Nova Tramandaí e Jardim do Éden.

Devido à esta carência de informações sobre as aves que frequentam o litoral norte do Rio Grande do Sul, principalmente em trechos de intensa ocupação antrópica, e a importância desse grupo como um constituinte da biodiversidade, o presente estudo apresenta um levantamento da avifauna costeira. Em um trecho compreendido entre o município de Tramandaí e Balneário Dunas Altas (município de Palmares do Sul), no litoral norte do Rio Grande do Sul, estabeleceuse a variação sazonal e estimou-se a abundância de cada espécie ao longo de um ano, de forma a fornecer dados que contribuam para a criação de estratégias conservacionistas deste grupo.

\section{Material e Métodos}

Para o presente trabalho entende-se como ambiente costeiro a área delimitada pela costa do mar e o começo 
das antedunas, conforme proposto por Naka e Rodrigues (2000). O estudo foi realizado no litoral norte do Rio Grande do Sul, entre o município de Tramandaí e Palmares do Sul, em uma extensão de $50 \mathrm{~km}$ de beira de praia, abrangendo os municípios de Tramandaí, Cidreira, Balneário Pinhal e Palmares do Sul (Figura 1).

Durante um período de 14 meses, com uma frequência de coleta de dados mensal e totalizando 23 $\mathrm{h}$ de observação, um trecho de $50 \mathrm{~km}$ foi percorrido com um automóvel, em velocidade média de $30 \mathrm{~km} / \mathrm{h}$.
Nos meses de verão (dezembro, janeiro e fevereiro), quando não foi possível trafegar pela beira da praia, foram estabelecidos seis pontos de observação, entre a barra do Rio Tramandaí (município de Tramandaí, limite norte) e o Farol Berta (município de Palmares do Sul, limite sul) (Tabela 1). Para cada ponto de observação préestabelecido foi considerado um campo de amostragem de $250 \mathrm{~m}$ para cada lado, com permanência de dois observadores por $30 \mathrm{~min}$. As observações de beira de praia iniciaram em março de 2010 e terminaram em abril de 2011, contemplando todas as estações do ano.

FIGURA 1: Mapas do Brasil e Rio Grande do Sul, com destaque para o trecho do litoral norte onde foi realizado o monitoramento da avifauna.

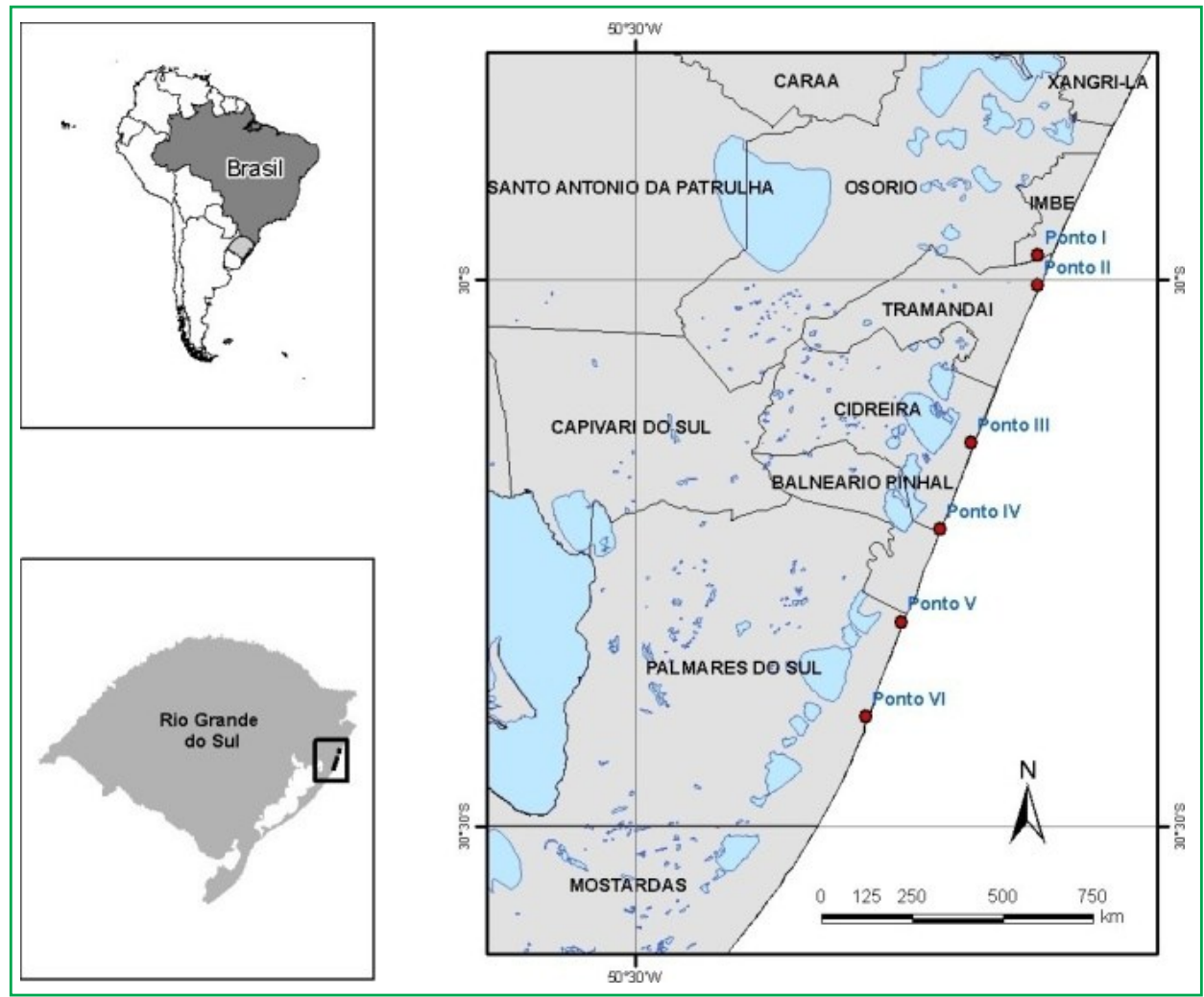

TABELA 1: Coordenadas geográficas dos pontos de observação.

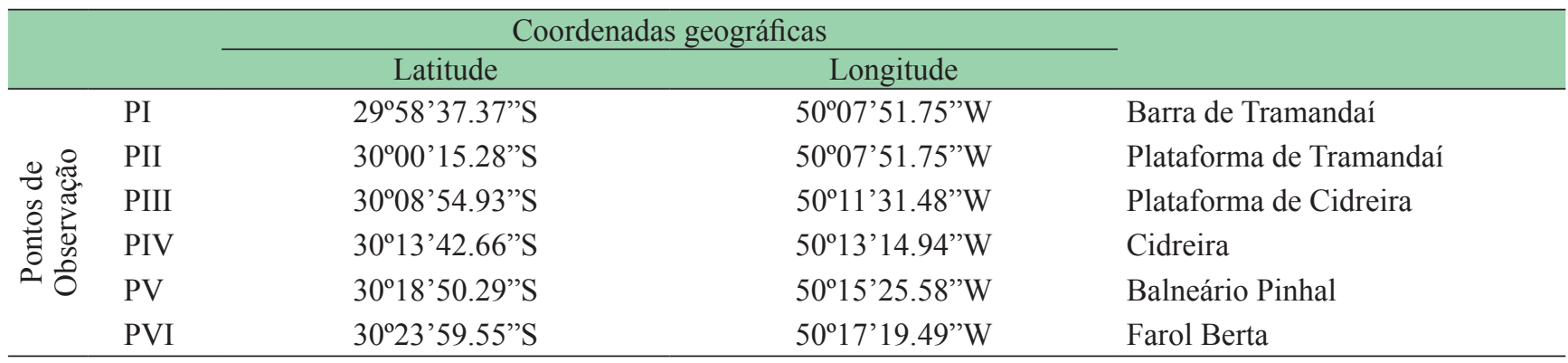


As aves presentes nesse trajeto (incluindo os Passeriformes), ocupando desde a zona de varrido, as dunas frontais e os sobrevoantes, foram observadas com auxílio de binóculo modelo BUSHNELL 10X50 e contabilizadas através de contagem direta do observador. Suas imagens, quando possível, foram capturadas com máquina fotográfica digital modelo SONY H-5. A nomenclatura e classificação das espécies estão de acordo com Bencke et al. (2010) e os indivíduos foram identificados com apoio de bibliografia específica (NOVELLI, 1997; BELTON, 2000; NAKA; RODRIGUES, 2000; NACINOVIC, 2005; OLMOS, 2009).

Os exemplares encontrados mortos foram contabilizados e incluídos nas análises, além de coletados e levados ao Laboratório de Zoologia da Universidade Feevale, quando em condições de conservação apropriadas.

\section{Análise de dados}

A frequência de ocorrência $(C)$ de cada espécie foi calculada através da equação $C=p \times 100 / P$, onde: " $p$ " corresponde ao número de contagens contendo a espécie e "P" o número total de contagens do período de estudo (14), sendo cada espécie considerada como: constante (presente em mais de 50\% das contagens), esporádica (presente em $25 \%$ a $50 \%$ das contagens) e acidental (presente em menos de $25 \%$ das contagens) (DAJOZ, 1983).

Para verificar como o número de espécies aumentou com o número de meses de observação foi construída uma curva de rarefação baseada na presença e ausência das espécies durante os meses, utilizando o programa estatístico EstimateS 8.0 (COLWELL, 2005), sendo que a estabilização da curva é considerada quando a mesma atinge uma assíntota. $\mathrm{O}$ mesmo programa foi utilizado para realizar as estimativas de riqueza específica e o intervalo de confiança (95\%) através do estimador não paramétrico Jackknife1.

\section{Resultados e Discussão}

Durante o período de amostragem, entre a barra de Tramandaí e o Farol Berta, foram registradas 21.888 aves, distribuídas em 44 espécies, pertencentes a 20 famílias e 10 ordens. Destas, 10 espécies são visitantes sazonais oriundas do hemisfério norte, três são visitantes sazonais oriundos do sul do continente, 31 são residentes (CBRO, 2011) (Tabela 2). As famílias mais representativas em número de espécies foram Ardeidae ( $(n=7)$, Sternidae $(n=6)$, Charadriidae $(n=6)$ e Scopolacidae $(n=6)$. Segundo Rossi-Wongtschowski et al. (2006), foram registradas mais de 100 espécies de aves associadas aos sistemas costeiros e marinhos brasileiros. Costa e Sander (2008) monitoraram o trajeto de $70 \mathrm{~km}$ entre os municípios de Imbé a Torres e registraram 57 espécies, distribuídas em 13 ordens, totalizando 29.065 indivíduos.

A curva de rarefação indica uma tendência à estabilização. O estimador Jackknifel apontou que 12 espécies ainda poderão ser observadas no local do presente estudo, indicando que $78 \%$ da riqueza específica foi amostrada. Essa estimativa de riqueza gerada não fornece previsão precisa do número real de espécies em uma comunidade, mas aponta o valor mínimo esperado (COLWELL et al., 2004) (Figura 2).

As populações de aves migratórias que frequentam a costa gaúcha apresentam flutuações quanto ao número de espécies e de indivíduos durante o ciclo migratório (TELINO-JÚNIOR et al., 2003). Algumas espécies, que não atingiram a maturidade reprodutiva, permanecem nas praias durante todo o ano, voltando somente às áreas de reprodução quando aptos a nidificarem (SICK, 1979). Entre os meses de março a julho e de setembro a novembro, houve um aumento esperado de espécies, visto que as espécies migratórias vindas do hemisfério norte ocupam a costa durante os meses de agosto e abril e as espécies vindas do hemisfério sul entre março e agosto (SICK, 1979), o que explica o acréscimo de espécies nesses meses. Durante o verão, entre dezembro e fevereiro, não houve incremento de nenhuma espécie, o que pode ser atribuído, possivelmente, à expressiva e intensa presença humana no local de alimentação e repouso dessas aves, como uma possível migração dessas 
TABELA 2: Lista de aves observadas entre a barra do Rio Tramandaí e o Farol Berta, município de Palmares do Sul, nos 14 meses de observação. $\mathrm{R}=$ Residente; $\mathrm{VN}=$ Visitante sazonal oriundo do hemisfério norte; $\mathrm{VS}$ = Visitante sazonal oriundo do sul do continente. Classificação conforme Bencke et al. (2010) e ocorrência conforme CBRO (2011).

\begin{tabular}{|c|c|c|}
\hline Nome do Táxon & Nome popular & $\begin{array}{l}\text { Status c } \\
\text { ocorrên }\end{array}$ \\
\hline \multicolumn{3}{|l|}{ Anseriformes Linnaeus, 1758} \\
\hline \multicolumn{3}{|l|}{ Anatidae Leach, 1820} \\
\hline Amazonetta brasiliensis (Gmelin, 1789) & marreca-pé-vermelho & $\mathrm{R}$ \\
\hline Dendrocygna viduata (Linnaeus, 1766) & marreca-piadeira & $\mathrm{R}$ \\
\hline \multicolumn{3}{|l|}{ Sphenisciformes Sharpe, 1891} \\
\hline \multicolumn{3}{|l|}{ Spheniscidae Bonaparte, 1831} \\
\hline Spheniscus magellanicus (J. R. Forster, 1781) & pinguim-de-magalhães & VS \\
\hline \multicolumn{3}{|l|}{ Procellariiformes Fürbringer, 1888} \\
\hline \multicolumn{3}{|l|}{ Diomedeidae Gray, 1840} \\
\hline Thalassarche chlororhynchos (Gmelin, 1789) & albatroz-de-nariz-amarelo & VS \\
\hline \multicolumn{3}{|l|}{ Suliformes } \\
\hline \multicolumn{3}{|l|}{ Fregatidae Degland \& Gerbe, 1867} \\
\hline Fregata magnificens (Mathews, 1914) & tesourão & $\mathrm{R}$ \\
\hline \multicolumn{3}{|l|}{ Phalacrocoracidae Reichenbach, 1849} \\
\hline Phalacrocorax brasilianus (Gmelin, 1789) & biguá & $\mathrm{R}$ \\
\hline \multicolumn{3}{|l|}{ Pelecaniformes Sharpe, 1891} \\
\hline \multicolumn{3}{|l|}{ Ardeidae Leach, 1820} \\
\hline Nycticorax nycticorax (Linnaeus, 1758) & savacu & $\mathrm{R}$ \\
\hline Butorides striata (Linnaeus, 1758) & socozinho & $\mathrm{R}$ \\
\hline Bubulcus ibis (Linnaeus, 1758) & garça-vaqueira & $\mathrm{R}$ \\
\hline Ardea cocoi ( Linnaeus, 1766) & garça-moura & $\mathrm{R}$ \\
\hline Ardea alba (Linnaeus, 1758) & garça-branca-grande & $\mathrm{R}$ \\
\hline Syrigma sibilatrix (Temminck, 1824) & maria-faceira & $\mathrm{R}$ \\
\hline Egretta thula (Molina, 1782) & garça-branca-pequena & $\mathrm{R}$ \\
\hline \multicolumn{3}{|l|}{ Threskiornithidae Poche, 1904} \\
\hline Phimosus infuscatus (Lichtenstein, 1823) & maçarico-de-cara-pelada & $\mathrm{R}$ \\
\hline \multicolumn{3}{|l|}{ Falconiformes Bonaparte, 1831} \\
\hline \multicolumn{3}{|l|}{ Falconidae Leach, 1820} \\
\hline Caracara plancus (Miller, 1777) & caracará & $\mathrm{R}$ \\
\hline Milvago chimango (Vieillot, 1816) & chimango & $\mathrm{R}$ \\
\hline \multicolumn{3}{|l|}{ Charadriiformes Huxley, 1867} \\
\hline \multicolumn{3}{|l|}{ Charadriidae Leach, 1820} \\
\hline Vanellus chilensis (Molina, 1782) & quero-quero & $\mathrm{R}$ \\
\hline Pluvialis dominica (Statius Muller, 1776) & batuiruçu & $\mathrm{VN}$ \\
\hline Pluvialis squatarola (Linnaeus, 1758) & batuiruçu-de-axila-preta & $\mathrm{VN}$ \\
\hline Charadrius semipalmatus (Bonaparte, 1825) & batuíra-de-bando & $\mathrm{VN}$ \\
\hline Charadrius collaris (Vieillot, 1818) & batuíra-de-coleira & $\mathrm{R}$ \\
\hline Charadrius modestus (Lichtenstein, 1823) & batuíra-de-peito-tijolo & VS \\
\hline \multicolumn{3}{|l|}{ Haematopodidae Bonaparte, 1838} \\
\hline Haematopus palliatus (Temminck, 1820) & piru-piru & $\mathrm{R}$ \\
\hline
\end{tabular}


Recurvirostridae Bonaparte, 1831

Himantopus melanurus (Vieillot, 1817)

$\begin{array}{cc}\text { pernilongo } & \mathrm{R} \\ \text { maçarico-de-perna-amarela } & \mathrm{VN} \\ \text { vira-pedra } & \mathrm{VN} \\ \text { maçarico-de-papo-vermelho } & \mathrm{VN} \\ \text { maçarico-branco } & \mathrm{VN} \\ \text { maçarico-miúdo } & \mathrm{VN} \\ \text { maçarico-de-sobre-branco } & \mathrm{VN} \\ & \\ \text { gaivota-maria-velha } & \mathrm{R} \\ \text { gaivotão } & \mathrm{R} \\ \text { trinta-reis-anão } & \mathrm{R} \\ \text { trinta-reis-boreal } & \mathrm{VN} \\ \text { trinta-reis-de-bico-vermelho } & \mathrm{R} \\ \text { trinta-reis-de-coroa-branca } & \mathrm{R} \\ \text { trinta-reis-de-bico-amarelo } & \mathrm{R} \\ \text { trinta-reis-real } & \mathrm{R} \\ \text { talha-mar } & \mathrm{R}\end{array}$

Scolopacidae Rafinesque, 1815

Tringa flavipes (Gmelin, 1789)

Arenaria interpres (Linnaeus, 1758)

Calidris canutus (Linnaeus, 1758)

Calidris alba (Pallas, 1764)

Calidris pusilla (Linnaeus, 1766)

Calidris fuscicollis (Vieillot, 1819)

Laridae Rafinesque, 1815

Chroicocephalus maculipennis (Lichtenstein, 1823)

Larus dominicanus (Lichtenstein, 1823)

\section{Sternidae}

Sternula superciliaris (Vieillot, 1819)

Sterna hirundo (Linnaeus, 1758)

Sterna hirundinacea (Lesson, 1831)

Sterna trudeaui (Audubon, 1838)

Thalasseus acuflavidus (Cabot, 1847)

Thalasseus maximus (Boddaert, 1783)

Rynchopidae Bonaparte, 1838

Rynchops niger (Linnaeus, 1758)

Columbiformes Latham, 1790

Columbidae Leach, 1820

Columba livia (Gmelin, 1789)

Strigiformes Wagler, 1830

Strigidae Leach, 1820

Athene cunicularia (Molina, 1782)

Passeriformes Linnaeus, 1758

Tyrannidae Vigors, 1825

Pitangus sulphuratus (Linnaeus, 1766)

\section{Hirundinidae}

Pygochelidon cyanoleuca (Vieillot, 1817)

Passeridae Rafinesque, 1815

Passer domesticus (Linnaeus, 1758)

pombo-doméstico

coruja-buraqueira

bem-te-vi

andorinha-pequena-de-casa

pardal
$\mathrm{R}$

$\mathrm{VN}$

$\mathrm{VN}$

$\mathrm{VN}$

$\mathrm{VN}$

$\mathrm{VN}$

$\mathrm{VN}$

$\mathrm{R}$

$\mathrm{R}$

$\mathrm{R}$

$\mathrm{VN}$

$\mathrm{R}$

$\mathrm{R}$

$\mathrm{R}$

$\mathrm{R}$

$\mathrm{R}$

$\mathrm{R}$

$\mathrm{R}$

$\mathrm{R}$

$\mathrm{R}$

$\mathrm{R}$ espécies para outras áreas. Além disso, a alteração na metodologia de levantamento das aves durante os meses de veraneio contribuiu para a redução considerável do número de espécies, pela diminuição do esforço amostral nesses meses. Notou-se uma concentração maior de indivíduos, apenas nos meses de verão, no trecho do ponto I ao ponto II (Tabela 3), onde existia uma barreira que limitava parcialmente o acesso de veranistas, comparado aos demais trechos observados, onde apenas era proibido o acesso de veículos automotores. No entanto, essa maior concentração nesse local ocorreu apenas nos meses de verão, momento em que houve a mudança no método amostral.

O estudo de Cornelius et al. (2001) apontou que a composição e a abundância de aves presentes na costa do Chile foi afetada pela presença de seres humanos na beira de praia durante todo o ano, porém, a maior interferência foi observada nos meses de veraneio, quando coincide 
FIGURA 2: Curva de rarefação das espécies de aves e o estimador de riqueza Jackknife1, entre a barra do Rio Tramandaí e o Farol Berta durante 14 meses.

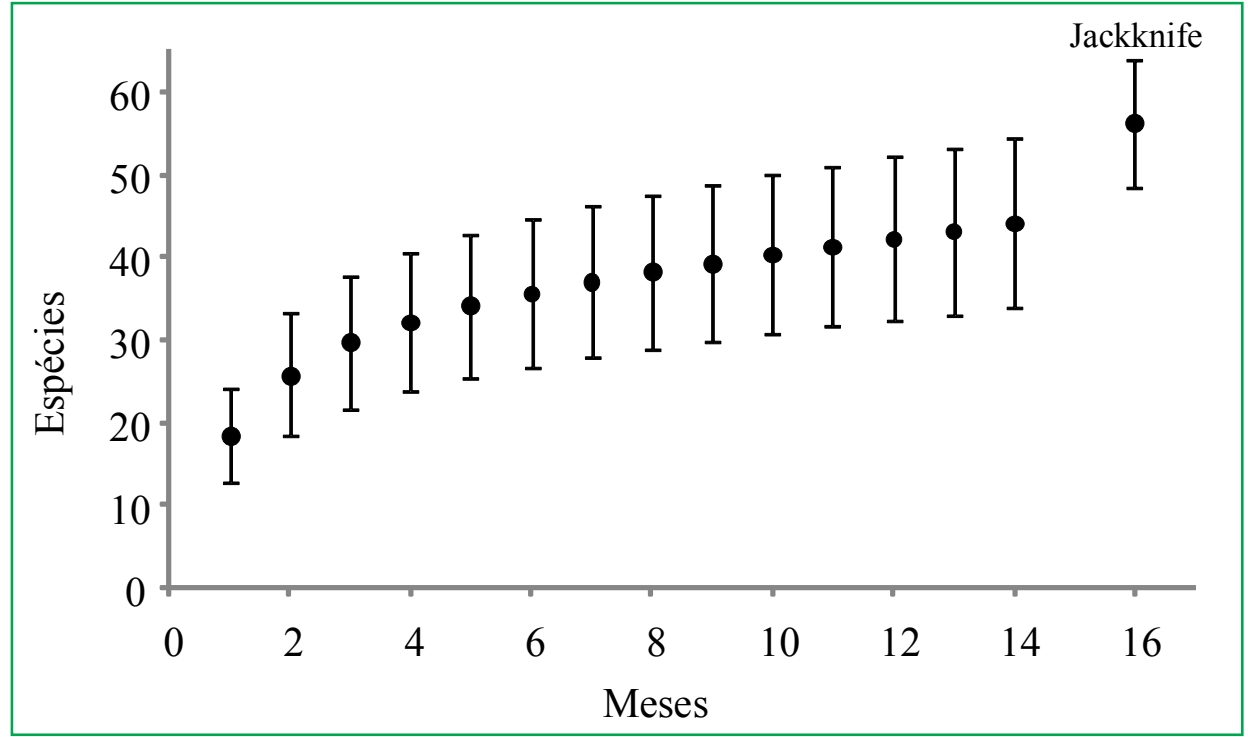

TABELA 3: Abundância mensal de aves nos trechos observados entre a barra do Rio Tramandaí e o Farol Berta, entre março de 2010 e abril de 2011.

\begin{tabular}{lrrrrrrrrrrrrrrr}
\hline \multicolumn{1}{c}{ Trecho } & marA & abrA & maio & jun & jul & ago & set & out & nov & dez & jan & fev & marB & abrB & Total \\
\hline PI - PII & 8 & 13 & 47 & 83 & 119 & 146 & 61 & 62 & 92 & 15 & 3 & 10 & 18 & 22 & 699 \\
PII - PIII & 964 & 1.239 & 581 & 389 & 382 & 360 & 569 & 540 & 511 & 8 & 1 & 1 & 1.691 & 2.048 & 9.284 \\
PIII - PIV & 37 & 201 & 318 & 167 & 178 & 91 & 179 & 108 & 36 & 1 & 1 & 1 & 363 & 263 & 1.944 \\
PIV - PV & 49 & 831 & 79 & 126 & 114 & 72 & 276 & 49 & 123 & 0 & 0 & 2 & 914 & 1.240 & 3.875 \\
PV - PVI & 133 & 1.313 & 507 & 201 & 197 & 209 & 387 & 228 & 107 & 0 & 0 & 1 & 1.320 & 1.483 & 6.086 \\
\hline
\end{tabular}

com a maior intensidade de pessoas na orla. No mesmo período, as aves foram avistadas em locais próximos que ofereciam recursos, sem interferência humana. Ikuta e Blumstein (2003) relataram que a presença humana pode afetar adversamente a abundância e o sucesso reprodutivo das aves. Algumas espécies diminuem a quantidade de tempo de forrageamento na presença de pessoas (LORD et al., 2001), a quantidade de cuidado parental aos filhotes quando perturbadas diminui (VERHULST et al., 2001) e aumenta a frequência cardíaca da ave em função do estresse (CULIK et al., 1990). Outros estudos demonstram que as aves evitam os seres humanos quando possível e preferem habitats menos perturbados (HOCKIN et al., 1992; BURGER et al., 1995; BURGER; GOCHFELD, 1998). Sendo assim, a presença de apenas sete espécies de aves costeiras e
44 indivíduos na estação de verão no presente estudo enfatizou a necessidade de conservação das áreas úmidas e lagoas próximas à costa gaúcha, já que estes locais oferecem refúgios para as aves principalmente nos meses em que a orla é mais antropizada.

$\mathrm{Na}$ primavera foram registrados representantes de 50 a $63 \%$ do total de espécies observadas no período estudado, enquanto no verão, apenas de 4 a $9 \%$. Para o outono foi registrado de 39 a $52 \%$ e para o inverno de 43 a 48\%. Quanto à frequência de ocorrência das espécies, foram observadas 19 espécies consideradas constantes, sete esporádicas e 18 espécies acidentais, o que indica que a maioria das espécies foi observada em apenas alguns meses, ao contrário de Scherer (2010) onde a maioria das espécies apresentou frequência de ocorrência constante. No presente estudo, o biguá, Phalacrocorax 
brasilianus, que é uma ave limícola e oportunista de amplo espectro trófico (BRANCO, 2000; LE LOUARN, 2003), foi observada em 13 meses e apresentou a maior frequência de ocorrência entre as aves $(\mathrm{C}=93 \%)$, como também observado em levantamentos da avifauna no litoral catarinense (SCHIEFLER; SOARES, 1994; BRANCO, 2000; BRANCO et al., 2004).

O mês de maior diversidade de aves costeiras foi outubro, contando com a presença de 29 espécies no trecho monitorado, embora o mês de maior abundância de espécies tenha sido abril a e b, somando 8.653 indivíduos (Tabela 4). Dessas ocorrências, a espécie mais abundante foi Calidris alba $(\mathrm{n}=5849)$, representante da família Scolopacidae, que teve expressiva presença no trajeto de estudo no mês de abril (a e b), que é, segundo Vooren e Chiaradia (1990), quando os indivíduos desta espécie que invernaram em hábitat lagunar utilizam o ambiente costeiro novamente como ponto de parada na sua migração ao norte (Tabela 4). A sua frequência de ocorrência esporádica $(\mathrm{C}=43 \%)$ indica que essa espécie concentrou-se em grande número de indivíduos em apenas alguns meses. Para Vooren e Brusque (1999), C. alba é uma das espécies que contribuíram de 43 a $66 \%$ ao número total de aves de todas as espécies na praia, nos meses de fevereiro a abril dos anos de 1982 a 1986 na Praia do Cassino, litoral sul do estado. Regionalmente e sazonalmente, as espécies de Scolopacidae que nidificam na América do Norte constituem a grande maioria das aves presentes na costa do Brasil, em abundância (VOOREN; BRUSQUE, 1999).

Das espécies encontradas mortas na beira da praia, sete exemplares $(\mathrm{C}=36 \%)$ são de Thalassarche chlororhynchos, ave marinha pelágica ameaçada de extinção (FONTANA et al., 2003), que ocorre mais comumente em alto-mar do que na costa marítima. As aves pelágicas são representadas por todos os Procellariiformes, sendo facilmente encontrados mortos na praia, sendo trazidos, geralmente, pelas correntes marinhas ou por tempestades em alto-mar (NAKA; RODRIGUES, 2000). Spheniscus magellanicus, pinguim-de-magalhães, classificado globalmente como ameaçado de extinção (quase ameaçado) de acordo com os critérios da IUCN (BIRDLIFE INTERNATIONAL, 2010), teve frequência de ocorrência esporádica $(\mathrm{C}=35 \%)$ e abundância de 341 indivíduos mortos. Os pinguins juvenis migram a partir do outono, auxiliados por correntes marinhas frias, como a corrente das Malvinas (SICK, 1997), em busca de regiões com maior disponibilidade de presas e a cada inverno muitos indivíduos alcançam a costa brasileira. Em geral, esses indivíduos se perdem dos seus grupos e podem sair da água em função do estado de debilidade física e/ou da baixa temperatura do corpo. As aves mortas nas praias do sul são sintomas da mortalidade natural das aves juvenis durante o inverno, mas há indícios de que em anos recentes a poluição por óleo vem causando mortalidade adicional (VOOREN; BRUSQUE, 1999).

TABELA 4: Diversidade e abundância das espécies de aves observadas entre a barra do Rio Tramandaí e o Farol Berta nos 14 meses de observação, entre março de 2010 e abril de 2011.

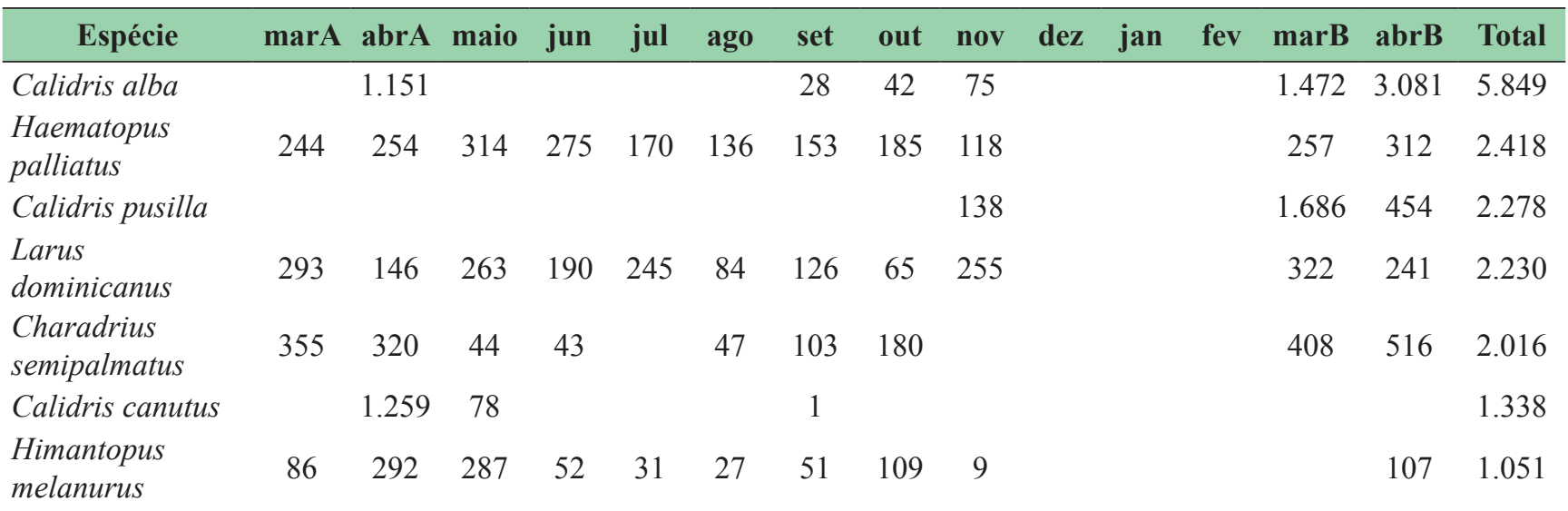




\begin{tabular}{|c|c|c|c|c|c|c|c|c|c|c|c|c|c|c|c|}
\hline Egretta thula & 40 & 43 & 91 & 32 & 46 & 54 & 67 & 25 & 60 & & & & 45 & 135 & 638 \\
\hline $\begin{array}{l}\text { Charadrius } \\
\text { collaris }\end{array}$ & 71 & 42 & 87 & 95 & 133 & 20 & 6 & 9 & 1 & & & & 59 & 45 & 568 \\
\hline Tringa flavipes & 30 & 10 & 20 & 10 & 7 & 42 & 216 & 166 & 7 & & & & & 31 & 539 \\
\hline $\begin{array}{l}\text { Chroicocephalus } \\
\text { maculipennis }\end{array}$ & 1 & 31 & 222 & 66 & 132 & 11 & & 3 & 1 & & & & 4 & 35 & 506 \\
\hline $\begin{array}{l}\text { Thalasseus } \\
\text { acuflavidus }\end{array}$ & & 5 & 1 & 3 & 30 & 25 & 351 & 8 & 5 & & & & & 3 & 431 \\
\hline $\begin{array}{l}\text { Spheniscus } \\
\text { magellanicus }\end{array}$ & & & & & 32 & 143 & 117 & 12 & 37 & & & & & & 341 \\
\hline Sterna trudeaui & & 1 & 71 & 102 & 42 & 55 & 41 & 2 & 4 & & 2 & & 2 & & 322 \\
\hline $\begin{array}{l}\text { Sterna } \\
\text { hirundinacea }\end{array}$ & & & & 6 & 43 & 90 & 67 & 4 & 10 & & & & 2 & 4 & 226 \\
\hline Sterna hirundo & 1 & 4 & & 10 & 30 & & 72 & 37 & 54 & & & 2 & 1 & 14 & 225 \\
\hline $\begin{array}{l}\text { Phalacrocorax } \\
\text { brasilianus }\end{array}$ & 7 & 6 & 6 & 34 & 2 & 52 & 30 & 30 & 19 & & 3 & 8 & 17 & 3 & 217 \\
\hline Vanellus chilensis & 34 & 18 & 9 & 4 & 4 & 9 & & 4 & 6 & 9 & & 2 & 15 & 49 & 163 \\
\hline $\begin{array}{l}\text { Pygochelidon } \\
\text { cyanoleuca }\end{array}$ & & & & & 18 & 15 & 2 & 18 & 7 & 2 & & 3 & 2 & 5 & 72 \\
\hline $\begin{array}{l}\text { Thalasseus } \\
\text { maximus }\end{array}$ & 1 & 1 & 1 & 6 & 10 & 17 & 17 & 4 & & & & & 8 & 4 & 69 \\
\hline $\begin{array}{l}\text { Pitangus } \\
\text { sulphuratus }\end{array}$ & 2 & 1 & 6 & 5 & 2 & 15 & 1 & 10 & 13 & & & & & 7 & 62 \\
\hline $\begin{array}{l}\text { Sterna } \\
\text { superciliaris }\end{array}$ & & & 7 & 10 & 2 & 21 & 11 & 5 & 4 & & & & & & 60 \\
\hline $\begin{array}{l}\text { Pluvialis } \\
\text { dominica }\end{array}$ & 9 & & & & & & & 8 & 41 & & & & & 1 & 59 \\
\hline $\begin{array}{l}\text { Calidris } \\
\text { fuscicollis }\end{array}$ & 16 & 3 & & & & & & 20 & & & & & & & 39 \\
\hline Columba livia & & 3 & 5 & & 7 & 9 & 3 & 3 & & & & & 3 & 3 & 36 \\
\hline Passer domesticus & & & & & & & & 17 & & & & & & & 17 \\
\hline Butorides striata & 1 & & & & & & & 3 & 1 & 10 & & & & & 15 \\
\hline $\begin{array}{l}\text { Dendrocygna } \\
\text { viduata }\end{array}$ & & & 15 & & & & & & & & & & & & 15 \\
\hline Bubulcus ibis & & & & 14 & & & & & & & & & & & 14 \\
\hline $\begin{array}{l}\text { Milvago } \\
\text { chimango }\end{array}$ & & 1 & & 2 & 4 & 3 & & 1 & & & & & 2 & 1 & 14 \\
\hline Arenaria interpres & & & & & & & & 11 & & & & & & & 11 \\
\hline $\begin{array}{l}\text { Thalassarche } \\
\text { chlororhynchos }\end{array}$ & & & 2 & & & 1 & 3 & 2 & 1 & & & & & & 9 \\
\hline $\begin{array}{l}\text { Athene } \\
\text { cunicularia }\end{array}$ & & & & & & & & & 3 & 3 & & & & 2 & 8 \\
\hline Rynchops niger & & 3 & & 3 & & & & & & & & & & 2 & 8 \\
\hline Caracara plancus & & 1 & & 1 & & 2 & 3 & & & & & & & & 7 \\
\hline $\begin{array}{l}\text { Nycticorax } \\
\text { nycticorax }\end{array}$ & & & & & & & & 4 & & & & & & & I \\
\hline $\begin{array}{l}\text { Amazonetta } \\
\text { brasiliensis }\end{array}$ & & & & 3 & & & & & & & & & & & \\
\hline Ardea alba & & & 2 & & & & & & & & & & & & \\
\hline
\end{tabular}


Fregata

magnificens

Syrigma sibilatrix

Pluvialis

squatarola

Charadrius

modestus

Ardea cocoi

1

Phimosus

infuscatus

\begin{tabular}{|c|c|c|c|c|c|c|c|c|c|c|c|c|c|c|c|}
\hline $\begin{array}{l}\text { Total indivíduos/ } \\
\text { mês }\end{array}$ & 1.191 & 3.597 & 1.532 & 966 & 990 & 878 & 1.472 & 987 & 869 & 24 & 5 & 15 & 4.306 & 5.056 & 21.888 \\
\hline Total espécie/mês & 16 & 23 & 21 & 22 & 20 & 22 & 24 & 29 & 23 & 4 & 2 & 4 & 18 & 24 & 44 \\
\hline
\end{tabular}

Calidris canutus foi avistado em abril A, maio e setembro, apresentando frequência de ocorrência acidental $(\mathrm{C}=21 \%)$. Juntamente com o bando, foi observado um indivíduo anilhado e com presença de bandeirolas de cor azul e laranja. De acordo com Pan American Shorebird Program (PASP) (2011), as bandeirolas de cor azul e laranja, visualizadas no indivíduo, representam os países Brasil e Argentina, respectivamente, indicando que esse indivíduo foi capturado nos dois países. A presença da espécie ( $\mathrm{n}=$ 1.250) em abril do primeiro ano de monitoramento e a ausência em abril do ano seguinte no presente estudo foi um fato também observado em outros estudos no litoral médio do estado, onde não foi registrado nenhum indivíduo de C. canutus na estação de outono (março, abril e maio) em 1997 e registrada a ocorrência de 71 espécimes em 1998 na mesma estação do ano, assim como em 2006 (sem registro) e em $2007(n=190)$ (MÄDER et al., 2011). No presente estudo, a ausência da espécie no segundo ano pode estar relacionada ao fato da espécie ter ocupado outra região da costa para realizar o seu condicionamento ou pela interrupção do censo no mês de abril. Algumas observações na Patagônia sugerem que a migração dos indivíduos que estão na Terra do Fogo, por um motivo que ainda não está claro, tem-se atrasado de uma a duas semanas desde o ano 2000, fazendo com que a chegada do maçarico em Bahía Delaware seja tardia (NILES et al., 2008), possivelmente retardando a chegada da espécie nos seus pontos de condicionamento.

A ausência de Haematopus palliatus na área de estudo durante o verão coincide com o período reprodutivo dessa espécie, que utiliza locais mais ao extremo sul da costa gaúcha, onde existem um maior número de dunas com presença de vegetação para nidificar (CANABARRO; FEDRIZZI, 2010), podendo este ser um fator responsável pela falta de registro dessa ave na estação. Larus dominicanus, que teve frequência constante $(\mathrm{C}=78 \%$ ), não foi observado entre dezembro e fevereiro na área do estudo, gerando uma flutuação na abundância, que pode ter sido desencadeada pelos intensos movimentos dessas aves entre vários locais em busca de alimento e descanso, como já verificado em outros estudos (BARBIERI, 2008). Áreas úmidas próximas, como o estuário e lagoas, devem ser monitoradas durante os meses em que a orla é mais antropizada para verificar a possível variação espacial de L. dominicanus, assim como das demais espécies, durante o verão.

No momento em que se tem conhecimento sobre a importância de um ambiente para a biodiversidade, normas podem ser estabelecidas para promover uma interação entre homem e os estuários de uma maneira mais equilibrada (MANOEL, 2009). Através de levantamentos de aves é possível propor alternativas de preservação e manejo do local que a avifauna utiliza para seu condicionamento físico, levando em consideração, ainda, o melhoramento das condições socioeconômicas e ambientais dos habitantes (MANOEL, 2009). Segundo Noess (1987), preservar espécies é preservar amostras representativas dos seus habitats.

O trânsito de veículos ocasiona um soterramento da biota encontrada no local através da compactação da 
areia, gerando uma barreira física para a alimentação das aves, conforme já observado por Vieira et al. (2004), para outras praias no Rio Grande do Sul. Outro fator preocupante para a preservação das praias é a degradação de dunas através da sua retirada e da ocupação irregular por edificações. Algumas estratégias de conservação do ambiente costeiro devem ser propostas a fim de minimizar o impacto ambiental já sofrido por essa área. Com o intuito de manter a diversidade de espécies de aves na beira da praia sugere-se a limitação ou mesmo a proibição do tráfego de veículos automotores, principalmente no mês de outubro, período em que foi observada a maior representatividade em número de espécies. Nos locais onde o tráfego já é proibido, como é o caso de parte da barra do rio Tramandaí, sugerese a manutenção deste bloqueio como estratégia de aumento da área de refúgio das aves no verão, uma vez que a margem pertencente ao município de Imbé encontra-se comprometida com estabelecimentos comerciais. Espera-se desta maneira que o estuário continue exercendo um papel importante no equilíbrio do meio ambiente por proporcionar condições para a manutenção de suas teias tróficas. Segundo o Projeto Dunas Costeiras - Conservação e Manejo (2011), as dunas são patrimônios comunitários de extrema relevância que exercem importantes funções ambientais, entre elas, servir de abrigo para nidificações de aves. A estratégia de manter as passarelas de acesso à praia e a implementação de novas, principalmente nos balneários ao sul de Tramandaí, além da desocupação das áreas invadidas, podem auxiliar na preservação das dunas.

Conciliando as estratégias propostas com atividades de conscientização da população que frequenta o litoral, e com uma fiscalização ativa pelas autoridades competentes, certamente auxiliará a mitigar os impactos antrópicos causados sobre o ecossistema costeiro e sua avifauna característica.

\section{Agradecimentos}

Ao pesquisador Ricardo Aranha Ramos pela elaboração da Figura 1 desse manuscrito.

\section{Referências}

ABSALONSEN, L.; TOLDO JUNIOR, E. E. T. A influência da inflexão costeira na variabilidade da linha de praia em Mostardas - RS. Revista Pesquisas em Geociências, Porto Alegre, v. 34, n. 1 p. 3-18, 2007.

ACCORDI, I. A. Ecologia e conservação de aves em ambientes costeiros do Rio Grande do Sul. 2008. 127 f. Tese (Doutorado em Ecologia) - Universidade Federal do Rio Grande do Sul, Porto Alegre. 2008

AZEVEDO JUNIOR, S. M.; LARRAZÁBAL, M. E.; PENA, O. Aves aquáticas de ambientes antrópicos (salinas) do Rio Grande do Norte, Brasil. In: BRANCO, J. O. (Org.). Aves marinhas insulares brasileiras: bioecologia e conservação. Itajaí: Editora da UNIVALE, 2004. p. 255-266.

BARBIERI, E. Variação sazonal do gaivotão (Larus dominicanus) durante o ano de 2005 no estuário de Cananeia-Iguape-Ilha Comprida, São Paulo, Brasil. Biota Neotropica, São Paulo, v. 8, n. 2, p. 97-102, 2008.

BELTON, W. Aves do Rio Grande do Sul - Distribuição e biologia. São Leopoldo: Ed Unisinos, 2000. 584 p.

BENCKE, G. A.; DIAS, R. A.; BUGONI, L.; AGNE, C. E.; FONTANA, C. S.; MAURÍCIO, G. N.; MACHADO, D. B. Revisão e atualização da lista das aves do Rio Grande do Sul, Brasil. Iheringia, Série Zoologia, Porto Alegre, v. 100, n. 4, p. 519-556, 2010

BIRDLIFE INTERNATIONAL. IUCN Red List. 2010. Disponível em: <http://www.birdlife.org> Acesso em: 15 mar. 2011.

BRACK, P. Vegetação e paisagem do litoral norte do Rio Grande do Sul: patrimônio desconhecido e ameaçado. In: ENCONTRO SOCIOAMBIENTAL DO LITORAL NORTE DO RS: ECOSSISTEMAS E SUSTENTABILIDADE, II, 2006, Imbé. Resumos... Imbé: CECLIMAR - UFRGS, 2006. p. 46-71.

BRANCO, J. O. Avifauna associada ao estuário do Saco da Fazenda, Itajaí, SC. Revista Brasileira de Zoologia, Curitiba, v. 17, n. 2, p. 387-394, 2000.

BRANCO, J. O.; MACHADO, I. F.; BOVENDORP, M. S. Avifauna associada a ambientes de influência marítima no litoral de Santa Catarina, Brasil. Revista Brasileira de Zoologia, Curitiba, v. 21, n. 3, p. 459-466, 2004.

BURGER, J.; GOCHFELD, M. Effects of ecotourists on bird behaviour at Loxahatchee National Wildlife Refuge, Florida. Environmental Conservation, Cambridge, v. 25, p. 13-21, 1998.

BURGER, J.; GOCHFIELD, M.; NILES, L. J. Ecotourism and birds in coastal New Jersey: contrasting responses of birds, tourists, and managers. Enviromnental Conservation, Cambridge, v. 22, p. $56-65,1995$.

CALliARI, L. R.; PEREIRA, P. S.; DE OLIVEIRA, A. O.; FIGUEIREDO, S. A. Variabilidade das dunas frontais no litoral norte e médio do Rio Grande do Sul, Brasil. Gravel, Porto Alegre, v. 3, n. 15, p. 15-30, 2005.

CANABARRO, P. L.; FEDRIZZI, C. E. Aspectos da reprodução do piru-piru Haematopus palliatus (Charadriiformes: Haematopodidae) na Praia do Hermenegildo, Rio Grande do Sul, Brasil. Revista Brasileira de Ornitologia, São Paulo, v. 18, n. 3, p. 249-255, 2010. 
CBRO - COMITE BRASILEIRO DE REGISTROS ORNITOLÓGICOS. Lista das aves do Brasil. 2011. Disponível em: <http:www.cbro.gov.br>. Acesso em: 23 abr. 2011 (Versão 25/01/2011).

COLWELL, R. K. Estimates: statistical estimation of species richness and shared species from samples. Versão 7.5. Guia de usuário e aplicativo. 2005. Disponível em: <http://viceroy.eeb. uconn.edu/EstimateS>. Acesso em: 2 jun. 2011.

COLWELL, R. K.; MAO, C. X.; CHANG, J. Interpolatin, extrapolatin, and comparing incidence-based species accumulation curve. Ecology, New York, v. 85, p. 2717-2727, 2004.

CORNELIUS, C.; NAVARRETE, S. A.; MARQUET, P. A. Effects of human activity on the Structure of Coastal Marine BIRD assemblages in Central Chile. Conservation Biology, v. 15, n. 5, p. 1396-1404, 2001.

COSTA, E. S.; CASTRO, A. G. S. Falconiiformes e Cathartiformes no litoral norte do Rio Grande do Sul, Brasil: Análise e Abundância. Biodiversidade Pampeana, Uruguaiana, v. 5, n. 1, p. 20-24, 2007.

COSTA, E. S.; SANDER, M. Variação sazonal de aves costeiras (Charadriiformes e Ciconiiformes) no litoral norte do Rio Grande do Sul, Brasil. Biodiversidade Pampeana, Uruguaiana, v. 6, n. 1, p. 3-8, 2008.

CULIK, B.; ADELUNG, D.; WOAKES, A. J. The effect of disturbance on the heart rate and behaviour of Adélie penguins (Pygoscelis adeliae) during the breeding season. In: KERRY, K. R.; HEMPEL, G. (Ed.). Antarctic ecosystems: ecological change and conservation. Berlin: Springer-Verlag, 1990. p. 177-182.

DAJOZ, R. Ecologia geral. Petrópolis: Ed. Vozes, 1983. 472 p.

EFE, M. A. 2004. Aves marinhas das ilhas do Espírito Santo. In: BRANCO, J. O. (Org.). Aves marinhas insulares brasileiras: bioecologia e conservação. Itajaí: Editora da UNIVALE, 2004. p. 101-118.

FONTANA, C. S.; BENCKE, G. A.; REIS, R. E. Livro vermelho da fauna ameaçada de extinção no Rio Grande do Sul. Porto Alegre: EDIPUCRS. 2003. 632 p.

FRANZ, I.; OTT, P. H.; MACHADO, R.; FAUSTO, I. V. Primeiros registros de Sula dactylatra Lesson, 1831 (Pelecaniformes: Sulidae) no estado do Rio Grande do Sul, Brasil. Revista Brasileira de Ornitologia, São Leopoldo v. 16, n. 2, p. 178-180, 2008.

GERCO. Programa de Gerenciamento Costeiro. 1998. Disponível em: <http:www.fepam.rs.gov.br>. Acesso em: 16 set. 2008.

HOCKIN, D.; OUNSTED, M.; GORMAN, M.; HILL, D.; KELLER, V.; BARKER, M. A. Examination of the effects of disturbance on birds with reference to its importance in ecological assessments. Journal of Environmental Management, Leiden, v. 36, p. 253-286, 1992.

IKUTA, L. A.; BLUMSTEIN, D. T. Do fences protect birds from human disturbance? Biological Conservation, Boston, v. 112, p. 447-452, 2003.

LE LOUARN, H. Qualitative and quantitative estimation of the great cormorant Phalacrocorax carbo diet. Cormorant Research Group Bulletin, Lelystad, v. 5, p. 26-30, 2003.

LORD, A.; WAAS, J. R.; INNES, J.; WHITTINGHAM, M. J. Effects of human approaches to nests of northern New Zealand dotterels. Biological Conservation, Boston v. 98, p. 233-240, 2001.
MÄDER, A.; PETRY, M. V.; EFE, M. A. Litoral médio do RS. In: VALENTE, R.; SILVA, J. M. C.; STRAUBE, F. C; NASCIMENTO, J. L. X (Ed.). Conservação de espécies migratórias neárticas no Brasil. Belém: Editora Conservação Internacional, 2011. p. 325-330.

MANOEL, F. C. Diversidade e abundância da assembléia de aves aquáticas no ecossistema Saco da Fazenda, Itajaí-SC. 2009. 61 f. Monografia (Conclusão de Curso em Oceanografia) Universidade do Vale do Itajaí, Itajaí. 2009.

MAURÍCIO, G. N.; DIAS, R. A. Áreas prioritárias para a conservação de espécies de aves ameaçadas de extinção no litoral Sul do Rio Grande do Sul, Brasil. In: SIMPÓSIO DE ÁREAS PROTEGIDAS, I, 2001, Pelotas. Anais... Pelotas: Universidade Católica de Pelotas, 2001. p. 190.

NACINOVIC, J. B. Aves marinhas na Bacia de Campos. Série Guias de Campo: Fauna Marinha da Bacia de Campos. Rio de Janeiro, GEMM Lagos. FIOCRUZ, 60p. 2005.

NAKA, L. N.; RODRIGUES, M. As aves da Ilha de Santa Catarina. Florianópolis: Ed. da UFSC, 2000. 294 p.

NILES, L. J.; SITTERS, H. P.; DEY, A. D.; ATKINSON, P. W.; BAKER, A. J.; CARMONA, R.; CLARK, K. E.; CLARK, N. A.; ESPOZ, C.; GONZALEZ, P. M.; HARRINGTON, B. A.; HERNANDEZ, D. E.; KALASZ, K. S.; MATUS, R.; MINTON, C. D. T.; MORRISON, R. I. G.; PECK, M. K.; PITTS, W. ROBINSON, R. A.; SERRANO, I. L. Update to the status of the red knot Calidris canutus in the Western Hemisphere. New Jersey: New Jersey Department of Environmental Protection, U.S. Fish \& Wildlife Service, 2008. 287 p.

NOESS, R. F. From plant communities to landscapes in conservation inventories: a look at the nature conservancy (USA). Biological Conservation, Boston, v. 41, p. 11-37, 1987.

NOVELLI, R. Aves marinhas costeiras do Brasil (Identificação e biologia). Porto Alegre: Cinco Continentes, 1997. 92 p.

OLMOS, A. Aves en el Uruguay y su distribucíon global. Montivideo: Ed. Tradinco, 2009. 520 p.

PAN AMERICAN SHOREBIRD PROGRAM (PASP). Country flag codes. 2011. Disponível em: <http:www.pwrc.usgs.gov/BBL/ homepage/paspflag.cfm>. Acesso em: 05 set. 2011.

PROJETO DUNAS COSTEIRAS. Conservação e Manejo. 2011. Disponível em: <http:www.nema-rs.org.br/pags/pop_dunas.html>. Acesso em: 13 jun. 2011.

RAMBO, B. Fisionomia do Rio Grande do Sul: ensaio de monografia natural. 3. ed. São Leopoldo: Ed. Unisinos, 2000. 473 p. ROSSI-WONGTSCHOWSKI, C. L. D. B.; VALENTIN, J. L.; JABLONSKI, S.; AMARAL, A. C. Z.; HAZIN, F. H. V.; ELROBRINI, M. Ambiente marinho. In: Programa REVIZEE (Ed.) Relatório executivo: avaliação do potencial sustentável de recursos vivos na Zona Econômica Exclusiva do Brasil. Brasília: Ministério do Meio Ambiente, 2006. p. 21-75.

SCHERER, A. Variação sazonal e a influência da estrutura da paisagem na ocorrência de Charadriidae e Scolopacidae no litoral do Rio Grande do Sul. Dissertação (Mestrado em Biologia) - Universidade do Vale do Rio dos Sinos, São Leopoldo, 2010. 69 p.

SCHIEFLER, A. F.; SOARES, M. Estudo comparativo da avifauna das praias de Navegantes e Laguna, Santa Catarina. Biotemas, Florianópolis, v. 7, n. 1 e 2, p. 31-45, 1994. 
SEELIGER, U.; ODEBRECHT, C.; CASTELlO, J. P. Os ecossistemas costeiro e marinho do extremo sul do Brasil. Rio Grande: Ecoscientia, 1998. 341 p.

SEMA - SECRETARIA DO MEIO AMBIENTE. Projeto RS biodiversidade. 2007. Disponível em: <http: www.biodiversidade. rs.gov.br>. Acesso em: 7 abr. 2010.

SICK, H. Migrações de aves no Brasil. Brasil Florestal, São Paulo, v. 9, p. 7-10, 1979.

SICK, H. Ornitologia brasileira. Rio de Janeiro, Nova Fronteira, 1997. $862 \mathrm{p}$.

TELINO-JÚNIOR, W. R.; AZEVEDO-JÚNIOR, S. M.; LYRANEVES, R. M. Censo de aves migratórias (Charadriidae, Scolopacidae e Laridae) na Coroa do Avião, Igarassu, Pernambuco, Brasil. Revista Brasileira de Zoologia, Curitiba, v. 20, n. 3, p. 451456, 2003.
VERHULST, S.; OOSTERBEEK, K.; ENS, B. J. Experimental evidence for effects of human disturbance on foraging and parental care in oystercatchers. Biological Conservation, Boston, v. 101, p. 375-380, 2001.

VIEIRA, H.; CALLIARI, L. J.; OLIVEIRA, G. P. O estudo do impacto da circulação de veículos motorizados na praia do Cassino através de parâmetros físicos. In: CONGRESSO DE PESQUISA E ENSINO EM TRANSPORTES, XVIII, 2004, Brasília. Anais... Brasília: UNB. 2004. Versão eletrônica.

VOOREN, C. M.; BRUSQUE, L. F. Aves de ambientes costeiros do Brasil: biodiversidade e conservação. 1999. Disponível em: $<$ http:www.bdt.org.br/workshop /costa/aves $>$. Acesso em: 12 abr. 2008.

VOOREN, C. M.; CHIARADIA, A. Seazonal abundance and behaviour of coastal birds on Cassino Beach, Brazil. Ornitologia Neotropical, Montreal, v. 1, p. 9-24, 1990. 